\title{
Radial Basis Function Neural Network Application to Measurement and Control of Shunt Reactor Overvoltages Based on Analytical Rules
}

\author{
Iman Sadeghkhani, ${ }^{1}$ Abbas Ketabi, ${ }^{2}$ and Rene Feuillet ${ }^{3}$ \\ ${ }^{1}$ Department of Electrical Engineering, Najafabad Branch, Islamic Azad University, \\ Najafabad 85141-43131, Iran \\ ${ }^{2}$ Department of Electrical Engineering, University of Kashan, Kashan 87317-51167, Iran \\ ${ }^{3}$ Grenoble Electrical Engineering Lab (G2ELab), Grenoble INP, BP46, \\ 38402 Saint Martin d'Hères Cedex, France \\ Correspondence should be addressed to Iman Sadeghkhani, i.sadeghkhani@ec.iut.ac.ir
}

Received 5 March 2012; Accepted 11 August 2012

Academic Editor: Gordon Huang

Copyright (C) 2012 Iman Sadeghkhani et al. This is an open access article distributed under the Creative Commons Attribution License, which permits unrestricted use, distribution, and reproduction in any medium, provided the original work is properly cited.

This paper presents an artificial intelligence application to measure switching overvoltages caused by shunt reactor energization by applying analytical rules. In a small power system that appears in an early stage of a black start of a power system, an overvoltage could be caused by core saturation on the energization of a reactor with residual flux. A radial basis function (RBF) neural network has been used to estimate the overvoltages due to reactor energization. Equivalent circuit parameters of network have been used as artificial neural network (ANN) inputs; thus, RBF neural network is applicable to every studied system. The developed ANN is trained with the worst case of the switching angle and remanent flux and tested for typical cases. The simulated results for a partial of 39-bus New England test system show that the proposed technique can measure the peak values and duration of switching overvoltages with good accuracy.

\section{Introduction}

In high-voltage (HV) power systems usually power is transmitted through long high-voltage transmission lines. During the low demand periods (nights or weekends), excessive reactive power produced by the capacitance of these lines causes a voltage increase over 1.1 p.u. at the high-voltage/medium-voltage (HV/MV) substations. For the absorption of the surplus reactive power, HV shunt reactors are connected to the receiving end of the transmission lines. Switching of those shunt reactors produce transients that need to be carefully studied and, if required, limited [1-7].

If the frequency characteristic of the system shows resonance conditions around multiples of the fundamental frequency, very high and weakly damped temporary 
overvoltages (TOVs) of long duration may occur when the system is excited by a harmonic disturbance [8-14].

Overvoltages can be classified as transient overvoltages, sustained overvoltages, harmonic resonance overvoltages, and overvoltages resulting from ferroresonance. Excessivesustained overvoltages may lead to the damage of transformers and other power system equipments. Transient overvoltages are a consequence of switching operations on long transmission lines, or the switching of capacitive devices, and may result in arrester failures. Ferroresonance is a nonharmonic resonance characterized by overvoltages whose waveforms are highly distorted and can cause catastrophic equipment damages [11, 15].

Overvoltage will put the shunt reactor into saturation, causing core heating and copious harmonic current generation. Circuit breaker called upon to operate during periods of high voltage will have reduced interrupting capability $[11,16]$.

In this paper power system blockset (PSB), a MATLAB/Simulink-based simulation tool $[17,18]$ is used for computation of temporary overvoltages. In order to study temporary overvoltages for a large number of possible system configurations, it is necessary to run many time-domain simulations resulting in a large amount of simulation time. A way to limit the overall calculation time is to reduce the number of simulations by applying analytical or knowledge-based rules to discard a number of system configurations before an actual timedomain simulation is carried out. This paper presents the artificial neural network (ANN) application for estimation of peak and duration overvoltages under switching transients during reactor energization. A tool such as the one proposed in this paper that can give the maximum switching overvoltage and its duration will be helpful to the operator during system restoration. Also it can be used as a training tool for the operators. Results of the studies are presented for a partial of 39-bus New England test system to illustrate the proposed approach.

\section{Study System Modelling}

The electrical components of the network are modeled using the MATLAB/Simulink environment [17]. These models should be adapted for the desired frequency range (here the frequencies up to $f=10 f_{0}$ are considered to be sufficient). The generator is represented by an ideal voltage source behind the subtransient inductance in series with the armature winding resistance that can be as accurate as the Park model [19]. Phase of voltage source is determined by the load flow results. Transmission lines are described by distributed line models. The circuit breaker is represented by an ideal switch. The shunt reactor model takes into account the leakage inductance as well as the magnetizing characteristics of the core, which is modeled by a resistance, $R_{m}$, simulating the core active losses and a saturable inductance, $L_{\text {sat }}$. The saturation characteristic is specified as a piecewise linear characteristic [20]. All of the loads are modeled as constant impedances.

\section{Harmonic Overvoltages during Restoration}

This paper concentrates on the estimation of harmonic overvoltages. These are a result of network resonance frequencies close to multiples of the fundamental frequency. They can be excited by harmonic sources such as saturated reactors, power electronics, and so forth. They may lead to long lasting overvoltages resulting in arrester failures and system faults [21, 22]. 


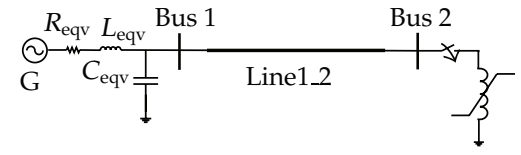

Figure 1: Sample system for shunt reactor energization study.

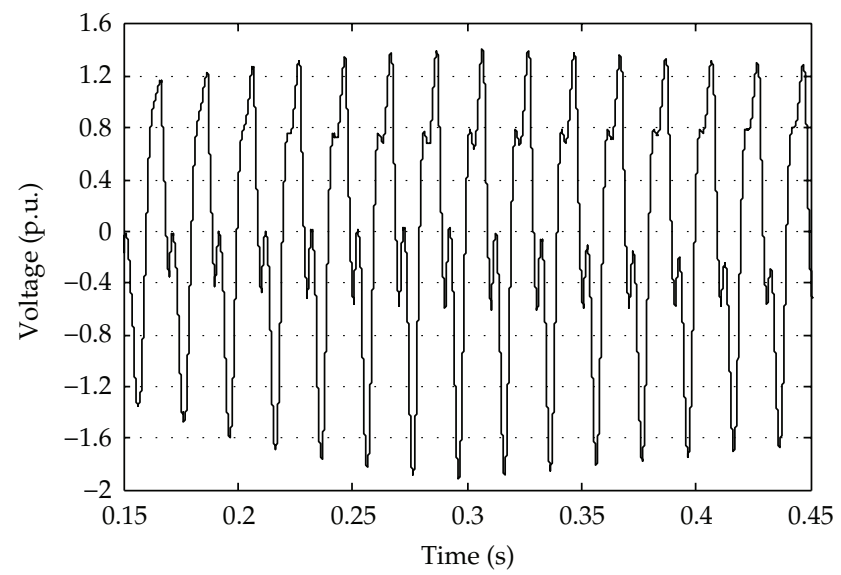

Figure 2: Voltage at bus 2 after switching of shunt reactor.

The sample system considered for explanation of the proposed methodology is a $400 \mathrm{kV}$ EHV network shown in Figure 1. The normal peak value of any phase voltage is $400 \sqrt{ } 2 / \sqrt{ } 3 \mathrm{kV}$ and this value is taken as base for voltage p.u. In the system studies $100 \mathrm{MVA}$ as a base power is considered. Figure 2 shows the switching transient at bus 2 when reactor is energized.

In practical system a number of factors affect the overvoltages factors due to energization or reclosing. In this paper, the following parameters are considered:

(i) voltage at shunt reactor bus before switching,

(ii) equivalent resistance of the network,

(iii) equivalent inductance of the network,

(iv) equivalent capacitance of the network,

(v) line length,

(vi) closing time of the circuit breaker poles,

(vii) shunt reactor capacity,

(viii) saturation curve slope; and

(ix) remanent flux.

In this paper, ANN training is based on Figure 1 that includes equivalent circuit parameters. In fact, ANN is trained just once for Figure 1. Therefore, it's possible to use 


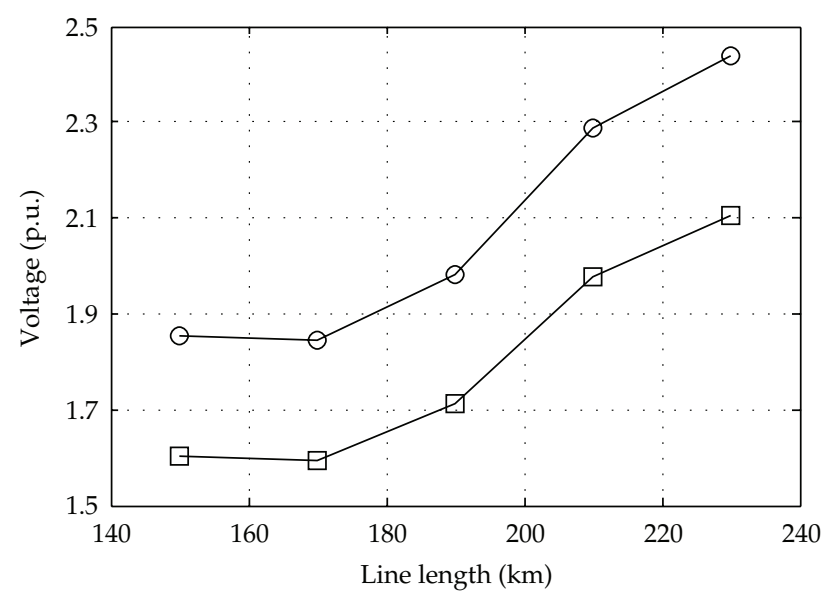

(a)

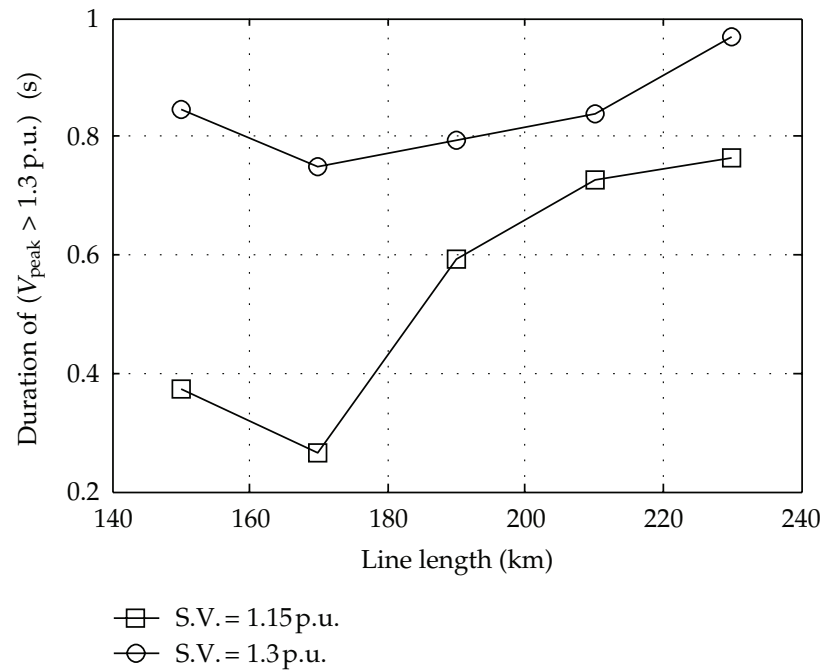

(b)

Figure 3: Overvoltage at bus 2 as line length while equivalent resistance 0.003 p.u., equivalent inductance 0.03 p.u., equivalent capacitance 1.282 p.u., switching angle $60^{\circ}$, shunt reactor capacity $20 \mathrm{MVAR}$, saturation curve slope 0.28 p.u., and remanent flux 0.8 p.u. S.V. is source voltage. (a) Peak; (b) duration.

developed ANN for estimation of overvoltages in every studied system. In Section 6 that developed ANN is tested for 39-bus New England test system, this issue is better understood. Also, a novel method based on worst case condition determination is proposed in Section 4 to reduce time-domain simulations.

Source voltage affects the overvoltage strongly. Figure 3 shows the effect of line length on overvoltage at different source voltage. Figure 4 shows the effect of saturation curve slope on overvoltages at different equivalent inductance. The saturation curve, and especially the $L_{\text {sat }}$, that is, the final slope of this curve, is a key point for the computation of the inrush currents. The reactor manufacturer provides a $L_{\text {sat }}$ slope value with a dispersion usually considered of $\pm 20 \%$. Figure 5 shows the effect of shunt reactor capacity on overvoltages at different equivalent resistance. Also, Figure 6 shows the effect of equivalent capacitance on overvoltages at different remanent flux. 


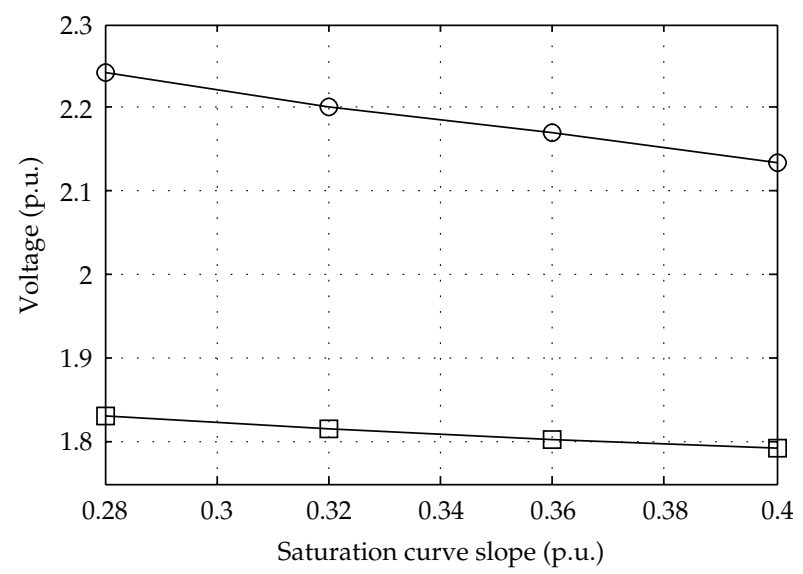

(a)

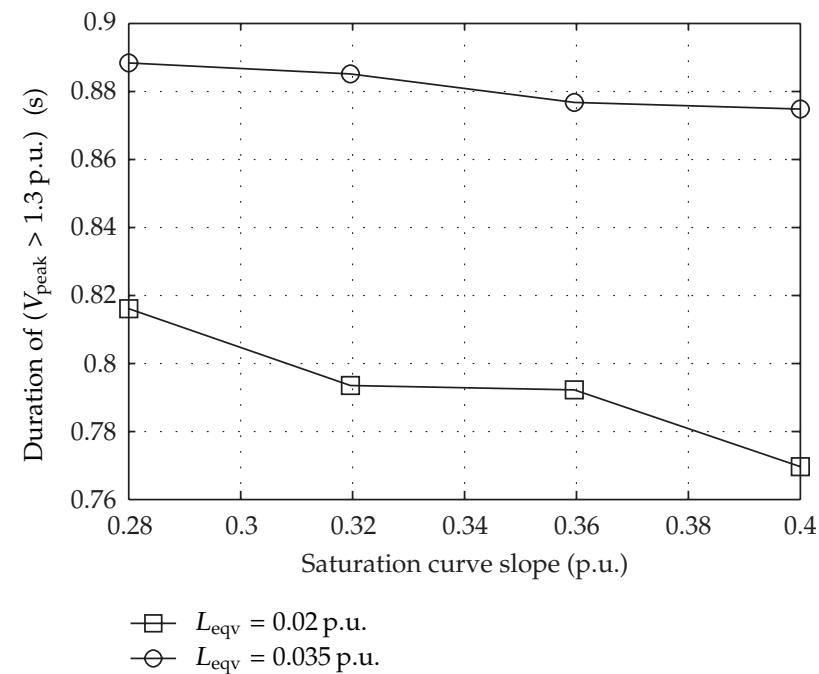

(b)

Figure 4: Overvoltage at bus 2 as saturation curve slope while source voltage 1.25 p.u, equivalent resistance 0.003 p.u., equivalent capacitance 1.282 p.u., line length $210 \mathrm{~km}$, switching angle $60^{\circ}$, shunt reactor capacity 20 MVAR, and remanent flux 0.8 p.u. $L_{\text {eqv }}$ is equivalent inductance. (a) Peak; (b) duration.

As discussed above for an existing system the main factors which affect the peak and duration values of switching overvoltage are voltage at reactor bus before switching, equivalent resistance, equivalent inductance, equivalent capacitance, line length, switching angle, reactor capacity, saturation curve slope, and remanent flux. Here it should be mentioned that a single parameter often cannot be regarded independently from the other important influencing factors.

The magnitude and duration of the overvoltages normally does not depend directly on any single-isolated parameter and a variation of one parameter can often alter the influence of another parameter, in other words, there exists an interaction between the various system and breaker parameters. This forbids the derivation of precise generalized rule of simple formulae applicable to all cases [23]. So an ANN can help to estimate the peak and duration values of switching overvoltages generated during reactor energization. An ANN is programmed 


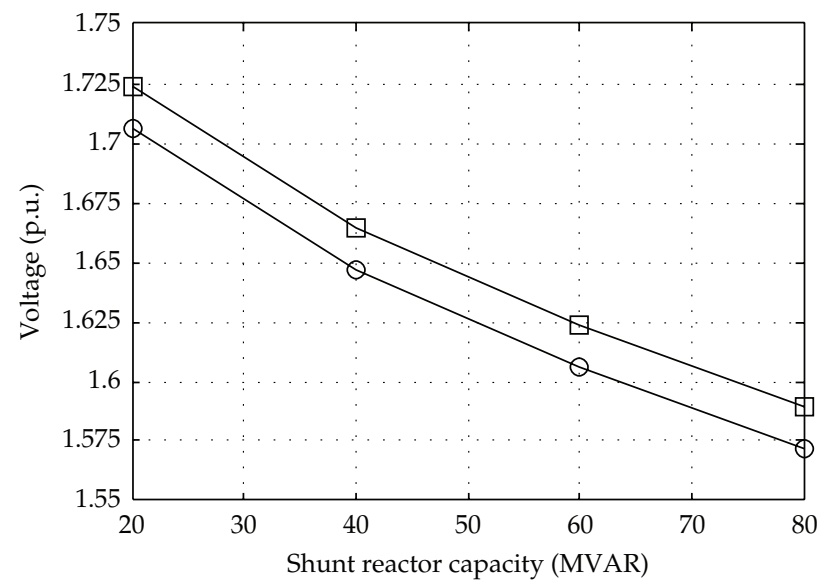

(a)

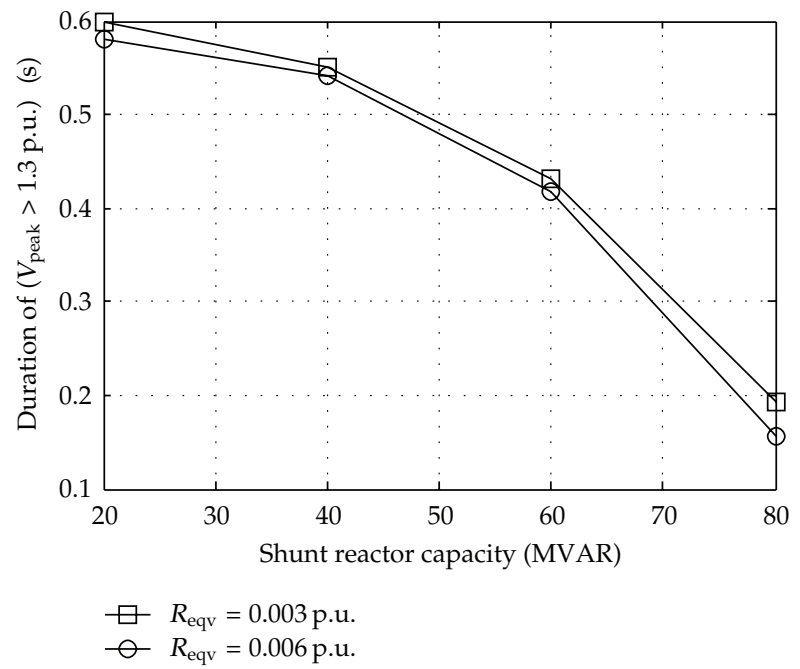

(b)

Figure 5: Overvoltage at bus 2 as shunt reactor capacity while source voltage 1.2 p.u., equivalent inductance 0.025 p.u., equivalent capacitance 1.8912 p.u., line length $190 \mathrm{~km}$, switching angle $45^{\circ}$, saturation curve slope 0.32 p.u., and remanent flux 0.8 p.u. $R_{\text {eqv }}$ is equivalent resistance. (a) Peak; (b) duration.

by presenting it with training set of input/output patterns from which it then learns the relationship between the inputs and outputs. In the next section, an ANN-based approach is described which can give an acceptable solution of switching transients by the help of which an operator can take a quick decision at the time of operation.

\section{Proposed Method for Harmonic Overvoltages Study}

\subsection{Worst-Case Condition Determination for Overvoltages Simulation}

Normally for harmonic overvoltages analysis, the worst case of the switching angle and remanent flux must be considered which it is a function of switching time, reactor 


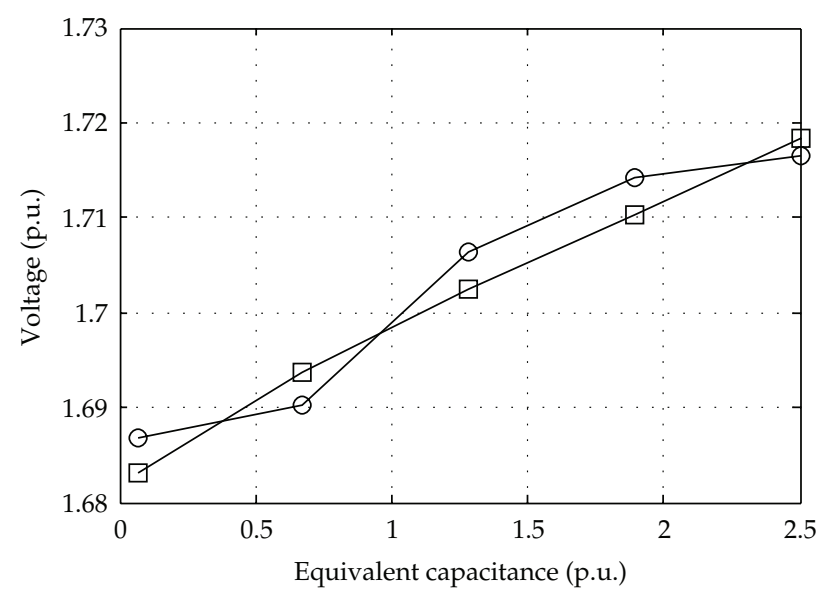

(a)

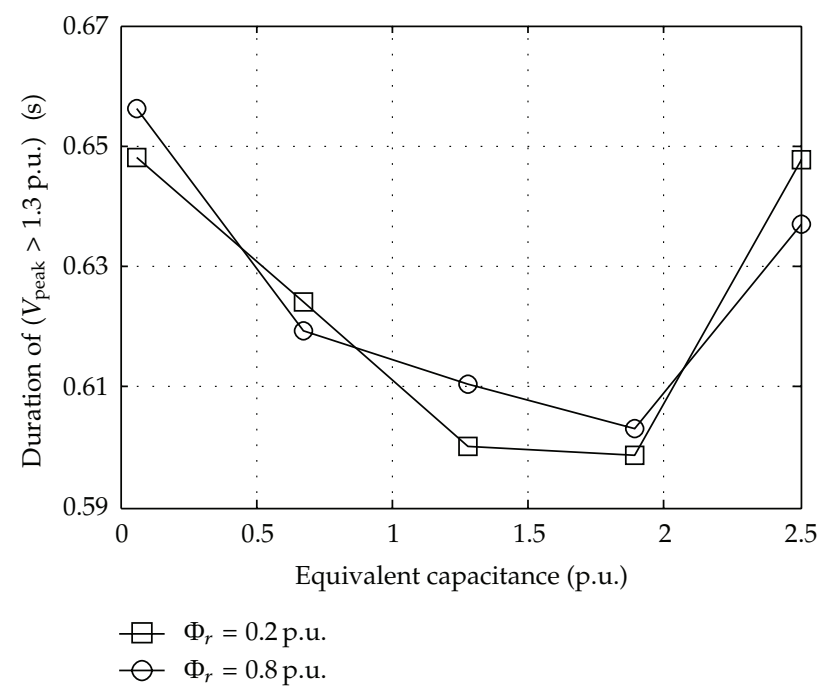

(b)

Figure 6: Overvoltage at bus 2 as equivalent capacitance while source voltage $1.2 \mathrm{p}$.u, equivalent resistance 0.004 p.u., equivalent inductance 0.025 p.u., line length $190 \mathrm{~km}$, switching angle $45^{\circ}$, shunt reactor capacity 20 MVAR, and saturation curve slope 0.32 p.u. $\Phi_{r}$ is remanent flux. (a) Peak; (b) duration.

characteristics and its initial flux condition, and impedance characteristics of the switching bus [20]. Using the worst switching angle and remanent flux, the number of simulations for each case can be reduced significantly.

In order to determine the worst-case switching time and remanent flux, the following index is defined as

$$
W=\sum_{h=2}^{10} Z_{j j}(h) \cdot I_{j}\left(h, t_{0}, \phi_{r}\right),
$$




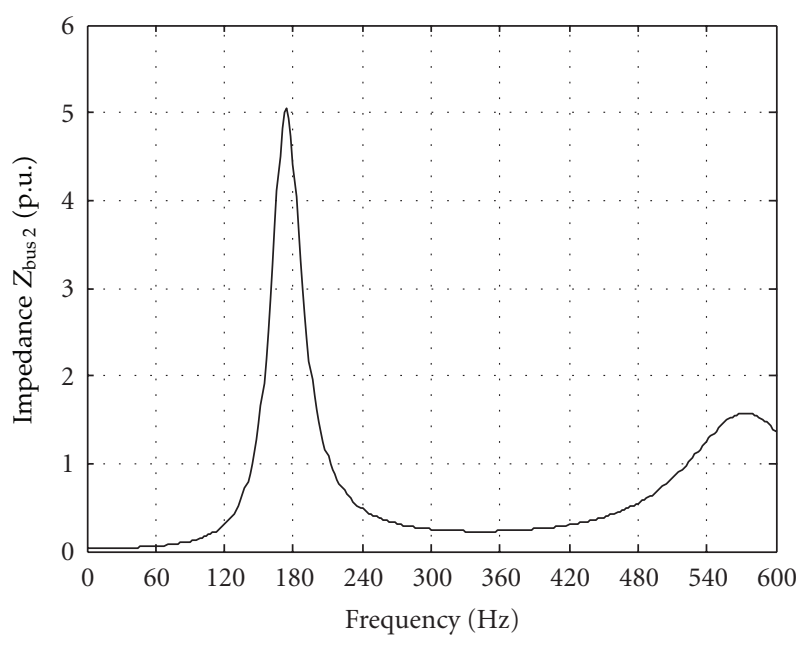

Figure 7: Voltage at bus 2 after switching of shunt reactor.

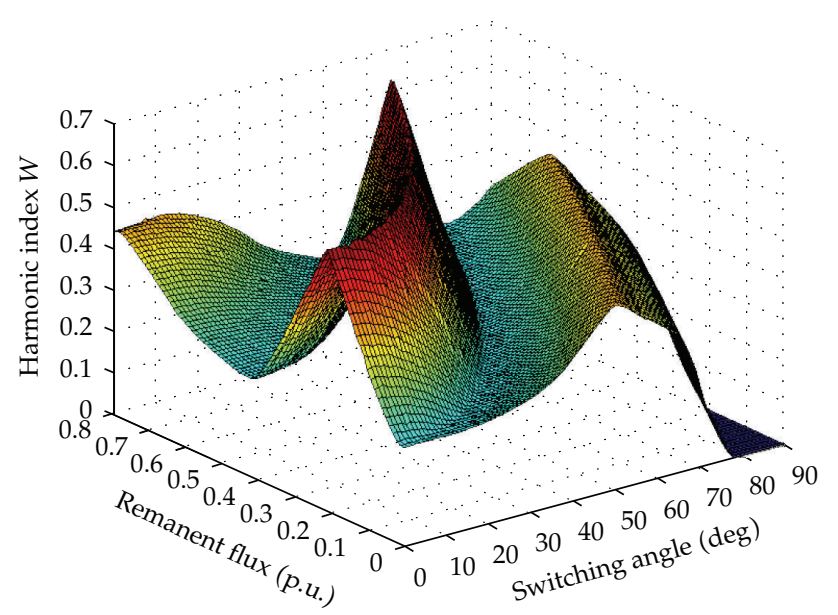

Figure 8: Changes of $W$ index with respect to current starting angle and remanent flux.

where $t_{0}$ is the switching time, $\phi_{r}$ is initial transformer flux, and $h$ is harmonic order. This index can be a definition for the worst-case switching angle and remanent flux. Using a numerical algorithm, one can find the switching time and remanent flux for which $W$ are maximal (i.e., harmonic overvoltages are maximal).

Figure 7 shows the result of the PSB frequency analysis at bus 2. The magnitude of the Thevenin impedance, seen from bus 2, Zbus2 shows a parallel resonance peak at $174 \mathrm{~Hz}$. Figure 8 shows changes of $W$ index with respect to the current starting angle and remanent flux. Figure 2 shows voltage at bus 2 after reactor switching for the worst-case condition (i.e., switching angle $20^{\circ}$ and remanent flux 0.27 p.u.). For temporary overvoltages, the overvoltage duration has to be taken into account in addition to the amplitude [24]. Table 1 summarizes the results of overvoltages simulation for four different switching angle and remanent flux that verify the effectiveness of $W$ index. 
Table 1: Effect of switching time and remanent flux on the maximum of overvoltages and duration of $V_{\text {peak }}>1.3$ p.u.

\begin{tabular}{lccc}
\hline Switching angle (deg.) & Remanent flux (p.u.) & $V_{\text {peak }}$ (p.u. $)$ & Duration of $\left(V_{\text {peak }}>1.3\right.$ p.u. $)(\mathrm{s})$ \\
\hline 20 & 0.27 & 1.9205 & 0.5628 \\
20 & 0.65 & 1.5841 & 0.3394 \\
75 & 0.27 & 1.6537 & 0.3064 \\
60 & 0.5 & 1.5293 & 0.2675 \\
\hline
\end{tabular}

\subsection{Steps of Assessment and Estimation of Switching Transient Overvoltages}

The steps for harmonic overvoltages assessment and estimation are as follows:

(1) determine the characteristics of shunt reactor that must be energized,

(2) calculate the $Z_{i i}(h)$ at the reactor bus for $h=2 f_{0}, \ldots, 10 f_{0}$,

(3) calculation of worst switching angle and remanent flux for simulation,

(4) run PSB simulation,

(5) calculation of overvoltage peak and duration,

(6) repetition of the above steps with various system parameters to learning artificial neural network, and

(7) testing of artificial neural network with different system parameters.

\section{The Radial Basis Function Neural Network (RBFNN)}

Figure 9 shows the structure of the RBF neural network, which comprises of three layers. The hidden layer possesses an array of neurons, referred to as the computing units. The number of such units can be varied depending on user's requirement $[25,26]$. Different basis functions like spline, multiquadratic, and Gaussian functions have been studied, but the most widely used one is the Gaussian type. In comparison to the other types of neural network used for pattern classification like back propagation feedforward networks, the RBF network requires less computation time for learning and has a more compact topology. The Gaussian RBF is found not only suitable in generalizing a global mapping but also in refining local features without altering the already learned mapping. Each hidden unit in the network has two parameters called a center $(\omega)$ and a width $(\sigma)$ associated with it. The response of one such hidden unit to the network input $\mathbf{X}, \mathbf{X}=\left[x_{1}, x_{2}, \ldots, x_{n}\right]^{T}$ is expressed as:

$$
\phi_{k}(\mathbf{X})=\exp \left(-\frac{1}{\sigma_{k}^{2}}\left\|\mathbf{X}-\omega_{k}\right\|^{2}\right)
$$

where $\omega_{k}$ is the center vector for $k$ th hidden unit, $\sigma k$ is the width of the Gaussian function, and || || denotes the Euclidean norm. The output layer comprises a number of nodes depending on the number of fault types to be classified which perform simple summation. The response of 


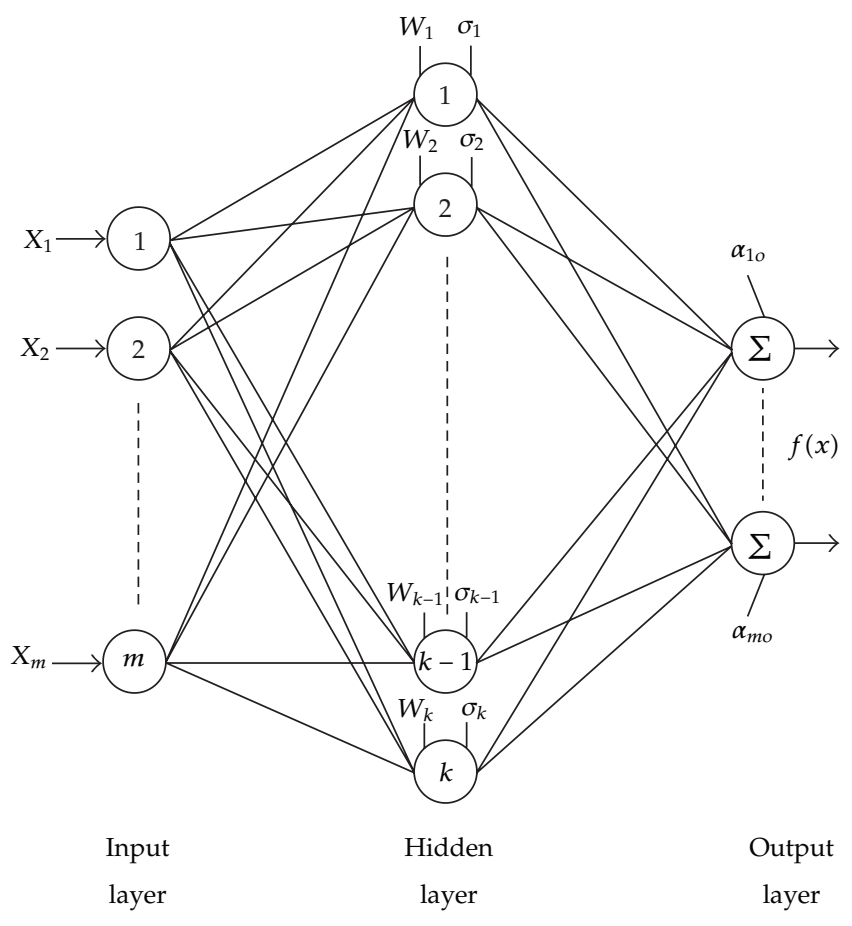

Figure 9: The structure of RBF neural network.

each hidden unit (4.1) is scaled by its connecting weights $(\alpha$ 's) to the output nodes and then summed to produce the overall network output. The overall network output is expressed as:

$$
f_{m}(\mathbf{X})=\alpha_{m o}+\sum_{k=1}^{N} \alpha_{m k} \phi_{k}(\mathbf{X})
$$

where $N$ indicates the total number of hidden neurons in the network, $\alpha_{m k}$ is the connecting weight of the $k$ th hidden unit to $m$ th output node, and $\alpha_{m o}$ is the bias term for the corresponding $m$ th output neuron. The learning process of the RBFNN involves with the allocation of new hidden units and tuning of network parameters. The learning process is terminated when the output error goes under the defined threshold [27].

\subsection{Training Artificial Neural Network}

All experiments have been repeated for different system parameters (2000 sets). 1000 sets were used to train RBFNN and 1000 sets were used to test RBFNN. RBFANN learned in 73 epochs. After learning, all parameters of the trained networks have been frozen and then used in the retrieval mode for testing the capabilities of the system on the data not used in learning. The testing data samples have been generated through the PSB program by placing the parameter values not used in learning, by applying different parameters. A large number 
Table 2: Some sample testing data and output.

\begin{tabular}{lcccccccccccc}
\hline$V$ & $R_{\text {eqv }}$ & $L_{\text {eqv }}$ & $C_{\text {eqv }}$ & L.L. & S.R. & $L_{\text {sat }}$ & $V_{\text {PSB }}$ & $V_{\text {RBF }}$ & error $_{V}$ & $T_{\text {PSB }}$ & $T_{\text {RBF }}$ & error $_{T}$ \\
\hline 1.208 & 0.003 & 0.0325 & 1.2825 & 180 & 20 & 0.34 & 1.6582 & 1.6793 & 1.2749 & 0.3673 & 0.3565 & 2.9517 \\
1.234 & 0.003 & 0.0325 & 1.2825 & 240 & 20 & 0.34 & 1.9675 & 1.9838 & 0.8261 & 0.4661 & 0.4741 & 1.7205 \\
1.257 & 0.003 & 0.0225 & 1.2825 & 220 & 20 & 0.3 & 1.8831 & 1.8657 & 0.9235 & 0.5154 & 0.5219 & 1.2694 \\
1.269 & 0.003 & 0.0275 & 1.2825 & 220 & 20 & 0.38 & 2.1371 & 2.2158 & 3.6829 & 0.8831 & 0.8692 & 1.5736 \\
1.292 & 0.003 & 0.0275 & 1.2825 & 160 & 20 & 0.26 & 1.8194 & 1.7917 & 1.5216 & 0.5747 & 0.5597 & 2.6128 \\
1.328 & 0.003 & 0.0275 & 1.2825 & 240 & 20 & 0.38 & 2.2339 & 2.1944 & 1.7684 & 0.9075 & 0.8968 & 1.1805 \\
1.354 & 0.003 & 0.0375 & 1.2825 & 180 & 20 & 0.34 & 2.0425 & 2.0850 & 2.0831 & 0.8573 & 0.8856 & 3.2985 \\
1.381 & 0.003 & 0.0325 & 1.2825 & 240 & 20 & 0.34 & 2.3117 & 2.3954 & 3.6194 & 0.9812 & 0.9958 & 1.4918 \\
1.296 & 0.0035 & 0.025 & 0.9781 & 190 & 10 & 0.32 & 1.8302 & 1.8234 & 0.3742 & 0.6035 & 0.6172 & 2.2657 \\
1.296 & 0.0035 & 0.025 & 0.9781 & 190 & 70 & 0.32 & 1.6653 & 1.6342 & 1.8671 & 0.5387 & 0.5235 & 2.8243 \\
1.301 & 0.0055 & 0.025 & 2.1956 & 190 & 50 & 0.32 & 1.7195 & 1.7059 & 0.7938 & 0.3995 & 0.4123 & 3.1984 \\
1.282 & 0.0065 & 0.025 & 1.5869 & 190 & 50 & 0.32 & 1.7102 & 1.6852 & 1.4628 & 0.3567 & 0.3645 & 2.1864 \\
1.294 & 0.0045 & 0.025 & 1.5869 & 190 & 90 & 0.32 & 1.6417 & 1.5735 & 4.1559 & 0.3126 & 0.3152 & 0.8215 \\
1.279 & 0.0045 & 0.025 & 0.3694 & 190 & 90 & 0.32 & 1.6215 & 1.6556 & 2.1035 & 0.2369 & 0.2408 & 1.6478 \\
1.306 & 0.0065 & 0.025 & 2.8044 & 190 & 30 & 0.32 & 1.7743 & 1.7253 & 2.7642 & 0.4734 & 0.4616 & 2.4961 \\
\hline
\end{tabular}

$V$ : voltage at shunt reactor bus before switching (p.u.), $R_{\text {eqv }}$ : equivalent resistance (p.u.), $L_{\text {eqv }}$ : equivalent inductance (p.u.), $C_{\text {eqv }}$ : equivalent capacitance (p.u.), L.L.: line length $(\mathrm{km})$, S.R.: shunt reactor capacity (MVAR), $L_{\text {sat }}$ : saturation curve slope (p.u.), error ${ }_{V}$ : voltage error (\%), and error $_{T}$ : duration time error (\%).

of testing data have been used to check the proposed solution in the most objective way at practically all possible parameters variation. Percentage error is calculated as:

$$
\operatorname{error}(\%)=\frac{|\mathrm{ANN}-\mathrm{PSB}|}{\mathrm{PSB}} \times 100
$$

Results for a sample test data are presented in Table 2. Values in column $V_{\mathrm{PSB}}$ are the absolute values of peak voltage at bus 2 calculated by PSB program in p.u. where the $V_{\mathrm{RBF}}$ values are the values simulated by trained network. Also values in column $T_{\mathrm{PSB}}$ are the values of overvoltage duration calculated by PSB program in second and $T_{\mathrm{RBF}}$ values are the values simulated by trained network.

The proposed model tested with portion of 39-bus New England test system. Various cases of shunt reactor energization are taken into account and corresponding peak and duration values estimated from trained model.

\section{Case Study}

In this section, the proposed algorithm is demonstrated for two case studies that are a portion of 39-bus New England test system, of which its parameters are listed in [28]. The simulations are undertaken on a single-phase representation. In the proposed method, first, studied system must be converted to equivalent circuit of Figure 1. In the other words, values of equivalent resistance, equivalent inductance, and equivalent capacitance are determined and used in trained artificial neural network to estimate overvoltages peak and duration.

Case 1. Figure 10 shows a one-line diagram of a portion of 39-bus New England test system which is in restorative state. The generator at bus 35 is a black-start unit. In order to reduce the 


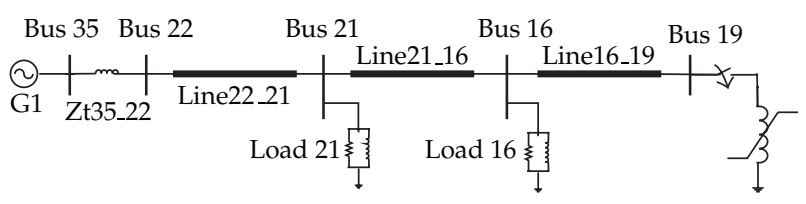

Figure 10: Studied system for Case 1.

Table 3: Case 1, some sample testing data and output.

\begin{tabular}{lcccccccc}
\hline$V$ & $\mathrm{~L} \mathrm{~L}$ & $\mathrm{~S} \mathrm{R}$ & $V_{\mathrm{PSB}}$ & $V_{\mathrm{RBF}}$ & error $_{V}$ & $T_{\mathrm{PSB}}$ & $T_{\mathrm{RBF}}$ & error $_{T}$ \\
\hline 1.1516 & 158 & 25 & 1.5844 & 1.5501 & 2.1672 & 0.2407 & 0.2486 & 3.2816 \\
1.1621 & 170 & 25 & 1.6162 & 1.6044 & 0.7314 & 0.2915 & 0.2878 & 1.2762 \\
1.2491 & 194 & 63 & 1.7104 & 1.7697 & 3.4694 & 0.4042 & 0.3851 & 4.7228 \\
1.2491 & 194 & 40 & 1.7567 & 1.7339 & 1.2951 & 0.4529 & 0.4637 & 2.3751 \\
1.3318 & 210 & 78 & 1.8975 & 1.8060 & 4.8216 & 0.5374 & 0.5522 & 2.7495 \\
1.3518 & 226 & 10 & 2.0649 & 2.1235 & 2.8357 & 0.6835 & 0.6601 & 3.4293 \\
1.4303 & 235 & 55 & 2.2143 & 2.2524 & 1.7224 & 0.7651 & 0.7529 & 1.5907 \\
1.4458 & 245 & 37 & 2.3096 & 2.3296 & 0.8639 & 0.9169 & 0.8906 & 2.8659 \\
\hline
\end{tabular}

$V$ : voltage at reactor bus before switching (p.u.), L L: line length $(\mathrm{km}), \mathrm{S}$ R: shunt reactor capacity (MVAR), error ${ }_{V}$ : voltage error $(\%)$, and error $_{T}$ : duration time error $(\%)$.

steady state overvoltage of no load transmission line, a reactor is connected at bus 19 . When the reactor is energized, harmonic overvoltages can be produced because of its nonlinear magnetization characteristics.

First, equivalent circuit of this system is determined and values of equivalent resistance, equivalent inductance, and equivalent capacitance are calculated, that is, this system is converted to system of Figure 1. In this case, values of equivalent resistance, equivalent inductance, and equivalent capacitance are 0.00291, 0.02427, and 2.474 p.u., respectively. For testing trained ANN, values of voltage at reactor bus (bus 19), line length, and shunt reactor capacity are varied and in each state, overvoltage peak and duration values are calculated from trained ANN and system of Figure 10. Table 3 contains some sample results of test data of Case 1.

Case 2. As another example, the system in Figure 11 is examined. In the next step of the restoration, unit at bus 6 must be restarted. In order to reduce the steady state overvoltage of no load transmission lines, the reactor at bus 6 should be energized. In this condition, harmonic overvoltages can be produced.

After converting this system to equivalent circuit of Figure 1, that is, after calculating equivalent circuit seen from bus 5, various cases of reactor energization are taken into account and corresponding overvoltages peak and duration are computed from PSB program and trained ANN. In this case, values of equivalent resistance, equivalent inductance, and equivalent capacitance are 0.00577, 0.02069, and 0.99 p.u., respectively. Summary of few results are presented in Table 4 . It can be seen from the results that the ANN is able to learn the pattern and give results to acceptable accuracy.

\section{Conclusion}

In this paper, a radial basis function-based method is presented to study switching overvoltages during shunt reactor energization. Also, a new approach is proposed to reduce 


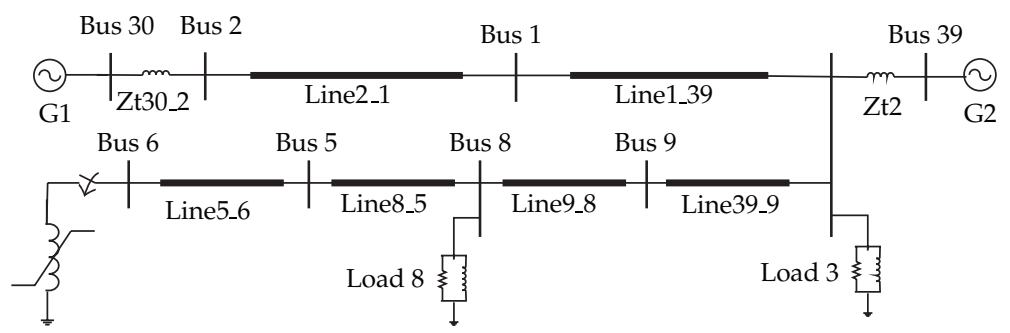

Figure 11: Studied system for Case 2.

Table 4: Case 2, some sample testing data and output.

\begin{tabular}{lcccccccc}
\hline$V$ & L L & S R & $V_{\text {PSB }}$ & $V_{\text {RBF }}$ & error $_{V}$ & $T_{\mathrm{PSB}}$ & $T_{\text {RBF }}$ & error $_{T}$ \\
\hline 1.1442 & 150 & 70 & 1.5011 & 1.5268 & 1.7135 & 0.1936 & 0.1881 & 2.8214 \\
1.1561 & 165 & 45 & 1.5453 & 1.5356 & 0.6281 & 0.2375 & 0.2268 & 4.5184 \\
1.2302 & 178 & 30 & 1.6769 & 1.6141 & 3.7428 & 0.3469 & 0.3557 & 2.5369 \\
1.2514 & 200 & 30 & 1.7481 & 1.7206 & 1.5746 & 0.3952 & 0.3902 & 1.2657 \\
1.3326 & 215 & 23 & 1.9507 & 1.9955 & 2.2974 & 0.5104 & 0.5214 & 2.1624 \\
1.3326 & 215 & 17 & 1.9914 & 1.9295 & 3.1108 & 0.5536 & 0.5612 & 1.3751 \\
1.4165 & 230 & 17 & 2.1652 & 2.2251 & 2.7659 & 0.6742 & 0.6407 & 4.9752 \\
1.4327 & 242 & 10 & 2.2479 & 2.2811 & 1.4782 & 0.7593 & 0.7809 & 2.8395 \\
\hline
\end{tabular}

$V$ : voltage at reactor bus before switching (p.u.), L L: line length $(\mathrm{km}), \mathrm{S}$ R: shunt reactor capacity (MVAR), error ${ }_{V}$ : voltage error $(\%)$, and error $_{T}$ : duration time error $(\%)$.

time-domain simulations based on worst switching angle and remanent flux determination. In addition, since equivalent circuit parameters of the network are used as ANN inputs, developed ANN is applicable to every studied system. The results from this scheme are close to results from the conventional method and helpful in predicting the overvoltage of the other case studies within the range of a training set. The proposed ANN approach is tested on a partial 39-bus New England test system. This method omits time-consuming time-domain simulations and it is suitable for real time applications during system restoration. Also it can be used as a training tool for the operators.

\section{References}

[1] C. D. Tsirekis, N. D. Hatziargyriou, and B. C. Papadias, "Control of shunt reactor inrush currents in the hellenic-interconnected power system," IEEE Transactions on Power Delivery, vol. 20, no. 2 I, pp. 757-764, 2005.

[2] X. Liu, X. Cui, and L. Qi, “Calculation of lightning-induced overvoltages on overhead lines based on DEPACT macromodel using circuit simulation software," IEEE Transactions on Electromagnetic Compatibility, vol. 54, no. 4, pp. 837-849, 2012.

[3] D. Li, C. Wang, and X. Liu, "Investigation of lightning-induced overvoltages affected by the sag of the overhead transmission line," in Proceedings of the IEEE Power Engineering and Automation Conference (PEAM), vol. 1, pp. 335-338, Wuhan, China, September 2011.

[4] Y. Shinohara, K. Fukui, S. Nomoto, and H. Matsubara, "Measurement of switching overvoltages at substations and on transmission lines in extra high voltage systems," in Proceedings of the Transmission and Distribution Exposition Conference: 2008 IEEE PES Powering Toward the Future (PIMS'08), pp. 1-6, Chicago, Ill, USA, April 2008.

[5] S. A. Taher and I. Sadeghkhani, "Estimation of magnitude and time duration of temporary overvoltages using ANN in transmission lines during power system restoration," Simulation Modelling Practice and Theory, vol. 18, no. 6, pp. 787-805, 2010. 
[6] P. Yang, S. Chen, and J. He, "Effect of different arresters on switching overvoltages in UHV transmission lines," Tsinghua Science and Technology, vol. 15, no. 3, pp. 325-328, 2010.

[7] S. Chen and H. Yu, "A review on overvoltages in microgrid," in Proceedings of the Asia-Pacific Power and Energy Engineering Conference (APPEEC'10), pp. 1-4, Chengdu, China, March 2010.

[8] B. Gustavsen, "Study of transformer resonant overvoltages caused by cable-transformer highfrequency interaction," IEEE Transactions on Power Delivery, vol. 25, no. 2, pp. 770-779, 2010.

[9] H. A. Hamid, N. Harid, and A. Haddad, "Determination of transient overvoltages during shunt reactor deenergization," in Proceedings of the 44th International Universities Power Engineering Conference (UPEC'09), pp. 1-4, Glasgow, UK, September 2009.

[10] A. Ketabi, I. Sadeghkhani, and R. Feuillet, "Using artificial neural network to analyze harmonic overvoltages during power system restoration," European Transactions on Electrical Power, vol. 21, no. 7, pp. 1941-1953, 2011.

[11] M. M. Adibi, R.W. Alexander, and B. Avramovic, "Overvoltage control during restoration," IEEE Transactions on Power Systems, vol. 7, no. 4, pp. 1464-1470, 1992.

[12] A. Ketabi, A. M. Ranjbar, and R. Feuillet, "Analysis and control of temporary overvoltages for automated restoration planning," IEEE Transactions on Power Delivery, vol. 17, no. 4, pp. 1121-1127, 2002.

[13] M. M. Adibi, J. N. Borkoski, and R. J. Kafka, "Analytical tool requirements for power system restoration," IEEE Transactions on Power Systems, vol. 9, no. 3, pp. 1582-1591, 1994.

[14] T. Hayashi, D. P. Roberts, K. Walve et al., "Modelling and simulation of Black Start and restoration of an electric power system. Results of a questionnaire," Electra, no. 131, pp. 155-169, 1990.

[15] I. Sadeghkhani and A. Ketabi, Switching Overvoltages during Restoration: Evaluation and Control Using ANN, Lambert Academic Publishing, 2012.

[16] D. Thukaram, H. P. Khincha, and S. Khandelwal, "Estimation of switching transient peak overvoltages during transmission line energization using artificial neural network," Electric Power Systems Research, vol. 76, no. 4, pp. 259-269, 2006.

[17] A. Ketabi and I. Sadeghkhani, Electric Power Systems Simulation Using MATLAB, Morsal Publications, 2011.

[18] G. Sybille, P. Brunelle, L. Hoang, L. A. Dessaint, and K. Al-Haddad, "Theory and applications of power system blockset, a MATLAB/Simulink-based simulation tool for power systems," in Proceedings of the IEEE Power Engineering Society Winter Meeting, pp. 774-779, 2000.

[19] M. M. Duro, "Damping modelling in transformer energization studies for system restoration: some standard models compared to field measurements," in Proceedings of the IEEE Bucharest PowerTech: Innovative Ideas Toward the Electrical Grid of the Future, pp. 1-8, Bucharest, Romania, July 2009.

[20] G. Sybille, M. M. Gavrilovic, J. Belanger, and V. Q. Do, "Transformer saturation effects on EHV system overvoltages," IEEE Transactions on Power Apparatus and Systems, vol. 104, no. 3, pp. 671-680, 1985.

[21] I. Sadeghkhani, A. Ketabi, and R. Feuillet, "Artificial neural network based method to mitigate temporary overvoltages," Journal of Engineering Science and Technology Review, vol. 4, no. 2, pp. 193200, 2011.

[22] I. Sadeghkhani, A. Ketabi, and R. Feuillet, "Study of transformer switching overvoltages during power system restoration using delta-bar-delta and directed random search algorithms," International Journal of Emerging Electric Power Systems, vol. 13, no. 3, pp. 1-22, 2012.

[23] Cigre Working Group, "Switching overvoltages in EHV and UHV systems with special reference to closing and reclosing transmission lines," Electra, no. 30, pp. 70-122, 1973.

[24] G. Morin, "Service restoration following a major failure on the hydroquebec power system," IEEE Transactions on Power Delivery, vol. 2, no. 2, pp. 454-463, 1987.

[25] S. Haykin, Neural Network: A Comprehensive Foundation, Prentice Hall, New York, NY, USA, 2nd edition, 1998.

[26] A. Karami and M. S. Mohammadi, "Radial basis function neural network for power system loadflow," International Journal of Electrical Power and Energy Systems, vol. 30, no. 1, pp. 60-66, 2008.

[27] P. K. Dash, A. K. Pradhan, and G. Panda, "Application of minimal radial basis function neural network to distance protection," IEEE Transactions on Power Delivery, vol. 16, no. 1, pp. 68-74, 2001.

[28] S. Wunderlich, M. M. Adibi, R. Fischl, and C. O. D. Nwankpa, "Approach to standing phase angle reduction," IEEE Transactions on Power Systems, vol. 9, no. 1, pp. 470-478, 1994. 


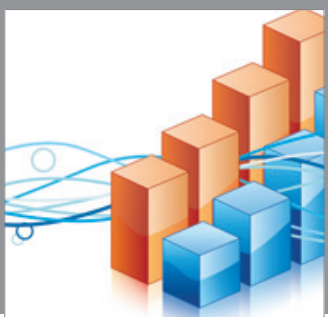

Advances in

Operations Research

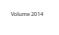

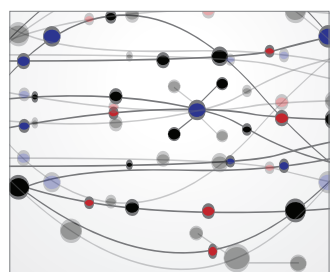

\section{The Scientific} World Journal
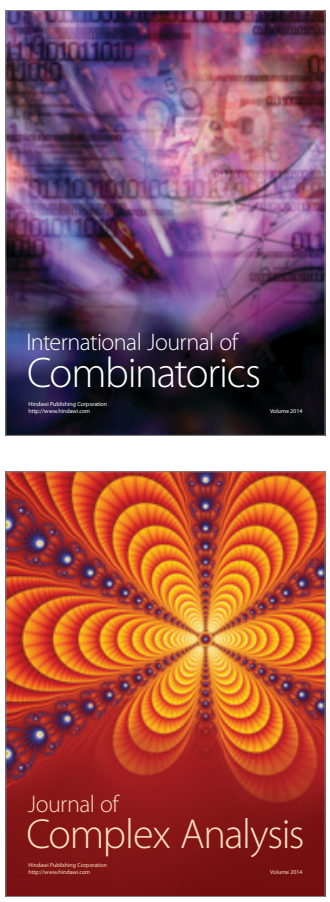

International Journal of

Mathematics and

Mathematical

Sciences
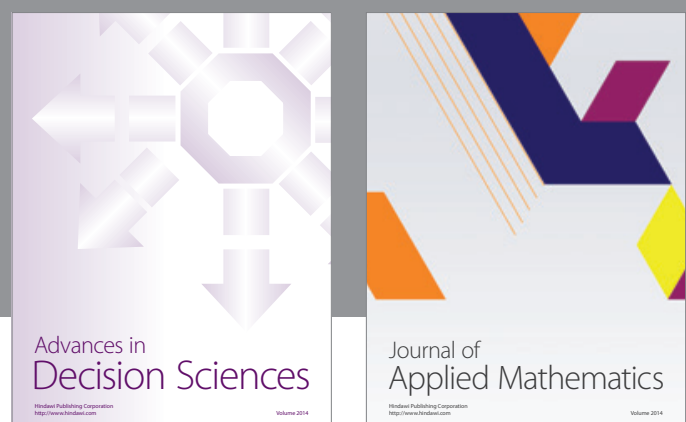

Journal of

Applied Mathematics
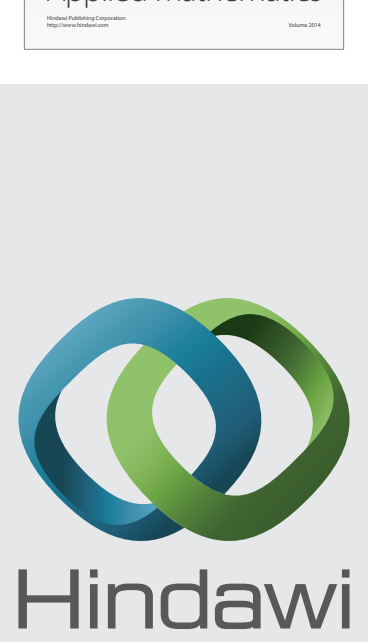

Submit your manuscripts at http://www.hindawi.com
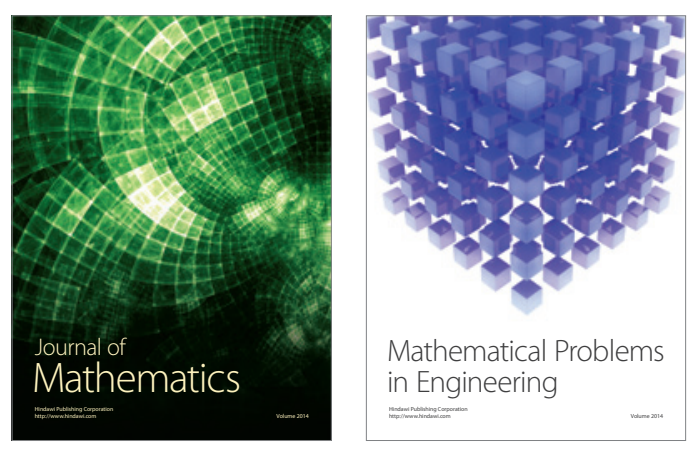

Mathematical Problems in Engineering
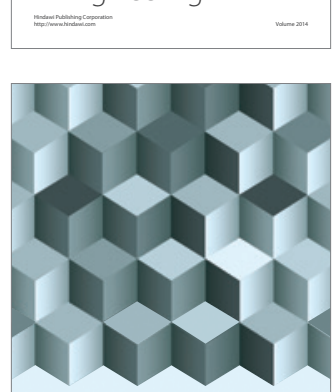

Journal of

Function Spaces
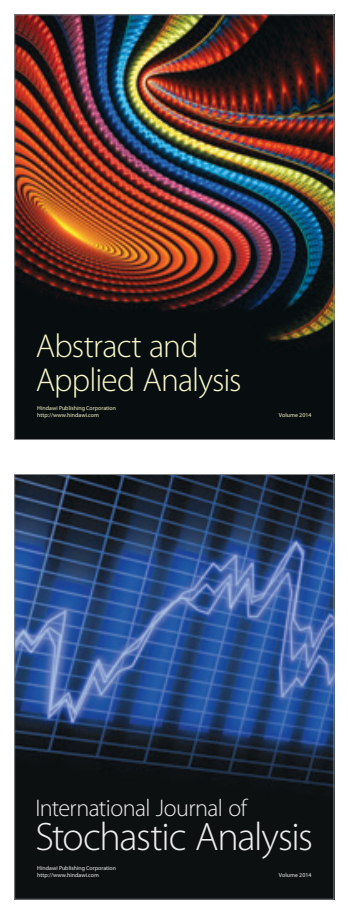

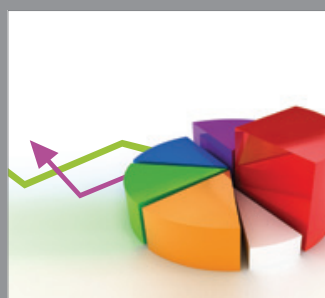

ournal of

Probability and Statistics

Promensencen
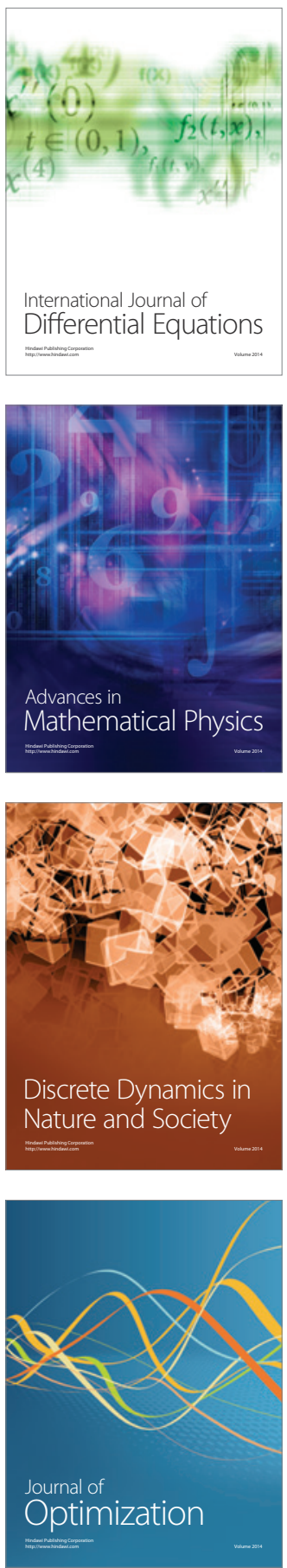